\title{
Matrix metalloproteinase pattern in celiac duodenal mucosa
}

\author{
Rachele Ciccocioppo ${ }^{1}$, Antonio Di Sabatino ${ }^{1}$, Michael Bauer ${ }^{2}$, Daniela N Della Riccia ${ }^{3}$, \\ Francesca Bizzini ${ }^{3}$, Federico Biagi ${ }^{1}$, Maria G Cifone $^{3}$, Gino R Corazza ${ }^{1}$ and \\ Detlef Schuppan ${ }^{2}$ \\ ${ }^{1}$ Gastroenterology Unit, IRCCS Policlinico San Matteo, University of Pavia, Italy; ${ }^{2}$ Department of Medicine I, \\ Friedrich-Alexander University of Erlangen-Nuremberg, Germany and ${ }^{3}$ Department of Experimental \\ Medicine, University of L'Aquila, Italy
}

\begin{abstract}
Matrix metalloproteinases (MMPs) are a family of endopeptidases playing a key role in tissue remodelling in both physiological and pathological conditions. Since little information is available about their role in celiac disease (CD), our aims were to quantify their expression/activity and to investigate their relation to proinflammatory cytokines in this condition. Duodenal biopsies from untreated, treated celiac patients and controls were used to quantify the expression of MMP-1, MMP-2, MMP-3, MMP-9, MMP-12, MMP-14, their inhibitor TIMP-1, IFN- $\gamma$ and TNF- $\alpha$ by using real-time reverse transcription-polymerase chain reaction and the gelatin/casein/elastin activities by gel zymography, and to isolate lamina propria mononuclear cells (LPMCs). These cells and myofibroblasts isolated from jejunal specimens were incubated in the absence or presence of IFN- $\gamma$ and TNF- $\alpha$. MMP-1 and MMP-12 mRNA levels were significantly increased in active CD compared to treated ( $P<0.01$ and $P<0.0005$, respectively) and normal mucosa $(P<0.01$ and $P<0.0005$, respectively), and this was paralleled by an upregulation of caseinolytic and elastolytic activities. Furthermore, MMP-12 levels significantly $(P<0.05)$ correlated with those of IFN- $\gamma$ and the degree of villous flattening. MMP-2 turned out to be significantly $(P<0.05)$ reduced in untreated and treated celiacs compared to controls. In active CD, transcripts of TIMP-1 were higher than in treated and controls $(P<0.005$ and $P<0.05$, respectively), such as those of IFN $-\gamma(P<0.05)$, whereas TNF- $\alpha$ levels were suppressed $(P=0.0001)$. In physiological condition, myofibroblasts represent the main source of MMP-2, whereas LPMCs produce almost all MMPs only after cytokine stimulation. Conversely, cells isolated from active patients constitutively express MMPs without any increase after cytokine stimulation, while those from treated patients are in a resting condition. In conclusion, our results show the presence of a peculiar MMP pattern in active CD strongly dominated by MMP-12, correlating either with IFN- $\gamma$ or the degree of mucosal damage.
\end{abstract}

Laboratory Investigation (2005) 85, 397-407, advance online publication, 20 December 2004; doi:10.1038/labinvest.3700225

Keywords: cytokines; celiac disease; extracellular matrix; metalloproteinases; mucosal damage

Celiac disease (CD) is an autoimmune enteropathy characterized by villous atrophy, crypt hyperplasia and heavy inflammatory infiltrate of both epithelium and lamina propria, caused by a T-cell response to an array of epitopes of dietary gliadin ${ }^{1,2}$ in genetically susceptible individuals ${ }^{3}$ carrying the HLA-DQ2 or -DQ8 phenotype. It is supposed that tissue transglutaminase, the CD autoantigen, ${ }^{4}$ increasingly released as a consequence of increasing tissue injury, ${ }^{5}$ catalyses gliadin deamidation and focuses T-cell response towards immunodominant

Correspondence: Professor GR Corazza, MD, Gastroenterology Unit, IRCCS Policlinico San Matteo, Piazzale Golgi, 5, 27100 Pavia, Italy.

E-mail: gr.corazza@smatteo.pv.it

Received 2 August 2004; revised 20 October 2004; accepted 23 October 2004; published online 20 December 2004 gliadin fragments. ${ }^{6}$ Elimination of gliadin and related prolamines from the diet allows restoration of mucosal integrity. Although there is evidence that activation of lamina propria $\mathrm{T}$ cells determines intestinal mucosal injury and remodelling, ${ }^{7}$ the final effector molecules involved in mucosal damage in CD are still poorly understood.

Matrix metalloproteinases (MMPs) are a family of calcium-activated, zinc-containing neutral endopeptidases mainly produced by stromal and immune cells, whose main function is the degradation of almost all components of the extracellular matrix (ECM) ${ }^{8,9}$ Even though a major role for MMPs in T-cell injury of the gut has already been shown in experimental models ${ }^{10}$ and some MMPs have been found raised in $C D,{ }^{11,12}$ a quantitative and wide assessment of MMPs in this pathological condition has never been performed. Similarly, the effect of 
the upregulation of $\mathrm{T}$ helper (Th)-1 cytokines ${ }^{13,14}$ on the activation of these enzymes has never been investigated in celiac mucosa.

This study was therefore carried out to evaluate the expression and the activity of several among the most known MMPs (MMP-1, MMP-2, MMP-3, MMP9, MMP-12, MMP-14) and their tissue inhibitor of metalloproteinase (TIMP)-1 by using real-time reverse transcription-polymerase chain reaction (RTPCR) assay and gel zymography in CD mucosa in comparison to normal mucosa, so as to investigate the putative correlation between MMP pattern and tissue damage. In addition, we proceeded to study the role of the two main proinflammatory Th-1 cytokines, interferon (IFN)- $\gamma$ and tumor necrosis factor (TNF)- $\alpha$, on their expression. Our findings support the idea that the hypersecretion of IFN- $\gamma$ is responsible for the activation of a particular MMP pattern strongly dominated by MMP-12, which appears to be a main contributor to the characteristic mucosal lesions of CD.

\section{Patients and methods}

\section{Patients and Biopsies}

Multiple endoscopic biopsy specimens were obtained from the second part of the duodenum from 11 untreated CD patients (eight females, mean age: 42.5 years, range: 18-66), 12 treated CD patients (nine females, mean age: 41.6 years, range: 28-66) and 10 consenting subjects (eight females, mean age: 42.6 years, range: 22-66) undergoing upper gastrointestinal endoscopy for functional dyspepsia. Subjects using steroidal or nonsteroidal anti-inflammatory drugs or presenting an inflamed mucosa at histology were excluded from the study.

For each patient and control, two well-oriented biopsies were processed according to standard methods for conventional histology and two biopsies were snap frozen at $-80^{\circ} \mathrm{C}$. Additional biopsies were also collected in culture medium to obtain a suspension of lamina propria mononuclear cells (LPMCs) as described below. All celiac patients were diagnosed on the basis of commonly accepted histological and serological criteria, namely the presence of subtotal villous atrophy with crypt hyperplasia and mucosal lymphocytic infiltration of the duodenal biopsy and positivity for antiendomysium/anti-tissue transglutaminase antibodies. In all untreated patients, a second biopsy, taken after a course of gluten-free diet, showed significant improvement of the lesion.

The study was approved by the local Ethics Committee and each patient gave informed consent to the study.

\section{RNA Preparation and RT-PCR}

Quantification of relative transcript levels of MMP-1, MMP-2, MMP-3, MMP-9, MMP-12, MMP-14,
TIMP-1, IFN- $\gamma$, TNF- $\alpha$, and the housekeeping gene $\beta 2$-microglobulin in the cDNA samples was performed by real-time quantitative RT-PCR using the TaqMan technology (PE-Biosystems, Foster City, CA, USA) on a LightCycler instrument (Roche, Mannheim, Germany). Supported by the Primer Express Software (Perkin-Elmer, Wellesley, MA, USA), specific primer and probe sets (see Table 1) for each transcript were generated by placing the plus and minus primer in separate exons and the $5^{\prime}$-carboxyfluorescein reporter dye and $3^{\prime}$-carboxy-tetramethylrhodamine (quencher) labelled probe on an exon-exon boundary in between. The inability to amplify genomic DNA was confirmed for each of the real-time RT-PCR systems by using genomic DNA as a template. Primer and probes were synthesized at MWG Biotech AG (Ebersberg, Germany). Real-time RT-PCR reactions were performed by using the LightCycler FastStart DNA Master Hybridization Probe Kit (Roche Molecular Biochemicals, Mannheim, Germany) according to the manufacturer's instructions. The final reaction mix included FastStart Taq DNA-Polymerase, dNTPs, $1 \times$ reaction buffer, $3.0 \mathrm{mM} \mathrm{MgCl}_{2}, 2 \mu \mathrm{M}$ of each primer, $0.5 \mu \mathrm{M}$ TaqMan probe and $1.5 \mu \mathrm{l}$ cDNA sample in a reaction volume of $15 \mu \mathrm{l}$. Generally RT-PCR samples were amplified with an initial Taq DNA polymerase activation step at $95^{\circ} \mathrm{C}$ for $10 \mathrm{~min}$, followed by 45 cycles of denaturation at $95^{\circ} \mathrm{C}$ for $0 \mathrm{~s}$, annealing at $60^{\circ} \mathrm{C}$ for $15 \mathrm{~s}$ and extension at $72^{\circ} \mathrm{C}$ for $6 \mathrm{~s}$. For IFN- $\gamma$ the annealing temperature was decreased to $53^{\circ} \mathrm{C}$ and extension was performed for $25 \mathrm{~s}$ at $65^{\circ} \mathrm{C}$. For analysis with the Proportional Second Derivative Maximum Option of the LightCycler Software (Roche, Version 3.5), a standard curve was generated by parallel amplification of 1:4-1:32 serial dilutions of a randomly chosen cDNA sample. To normalize for variations in RNA amounts and reverse transcription efficacies, transcript levels of the housekeeping gene $\beta 2$-microglobulin were determined. Relative transcript levels of cytokines or ECM proteins are expressed as the ratio between the gene of interest and $\beta 2$-microglobulin mRNA levels of the sample.

\section{Substrate Gel Electrophoresis (Zymography)}

One snap-frozen biopsy from each patient and control was thawed, homogenized in extraction buffer $\left(50 \mathrm{mM}\right.$ Tris- $\mathrm{HCl}$ at $\mathrm{pH} 7.4,10 \mathrm{mM} \mathrm{CaCl}_{2}$, $0.05 \%$ Brij 35, 0.25\% Triton X-100, $10 \mu \mathrm{g} / \mathrm{ml}$ trypsin, $10 \mu \mathrm{g} / \mathrm{ml}$ leupeptin, $1 \mathrm{mM}$ phenylmethanesulphonyl fluoride, $5 \mu \mathrm{g} / \mathrm{ml}$ aprotinin and $10 \mu \mathrm{g} / \mathrm{ml}$ pepstatin A) and sonicated. After centrifugation, the protein concentration was determined from the supernatants (Pierce BCA, Rockford, IL, USA), which were stored at $-80^{\circ} \mathrm{C}$ until required. Sodium dodecyl sulphate polyacrylamide gel electrophoresis zymograms containing $0.1 \%$ gelatin (Sigma Chemical Co., St Louis, MO, USA), $0.1 \%$ casein (Sigma Chemical 
Co.) or $0.1 \%$ elastin (Sigma Chemical Co.) were performed as previously described ${ }^{15}$ using 7-20-20 $\mu \mathrm{g}$ of sample proteins to reveal the gelanolyticcaseinolytic-elastolytic activities, respectively. Following electrophoresis, gels were rinsed in $50 \mathrm{mM}$ Tris-HCl (pH 7.4) containing $2 \%$ Triton $\mathrm{X}-100$ followed by $50 \mathrm{mM}$ Tris-HCl $(\mathrm{pH} 7.4)$ and incubated overnight for gelatin gels, or $72 \mathrm{~h}$ for casein and elastin gels, in a buffer containing $50 \mathrm{mM}$ Tris- $\mathrm{HCl}$ (pH 7.4), 0.2 M NaCl, $5 \mathrm{mM} \mathrm{CaCl}_{2}$, $1 \%$ Triton $\mathrm{X}-100$ at $37^{\circ} \mathrm{C}$. Enzyme expression was revealed by staining with $0.1 \%$ Coomassie blue in a mixture of acetic acid:methanol:water (1:3:6), and destaining in the same mixture without dye. All experiments were carried out in duplicate with or without $1.5 \mathrm{mM}$ 1,10-phenantroline (Sigma), a zincchelator that inhibits all MMP activities. Relative molecular weights $(\mathrm{Mw})$ of bands were analysed in comparison to the Mw of a standard (Novex Inc., San Diego, CA, USA) and the following standards were used as positive controls of MMPs: MMP-1 (Calbiochem, Biosciences Inc., La Jolla, CA, USA \#444208-Mw, $55 \mathrm{kDa}$ ), MMP-2 (Calbiochem; \#444213-Mw, 72 kDa), MMP-3 (Oncogene, Boston, MA, USA; \#PF063-Mw, $57 \mathrm{kDa}$ ), MMP-9 (Calbiochem; \#444231-Mw, $92 \mathrm{kDa}$ ) and for MMP-12 (Mw, $52 \mathrm{kDa}$ ) the supernatant of human macrophages cultured in the presence of granulocyte macrophage colony-stimulating factor (PeproTech EC Ltd, London, UK). Bands were quantified by scanning densitometry, using an LKB Ultrascan XL Laser Densitometer (Kodak Ltd., Hemel Hempstead, UK), as measurement of both intensity and area of each band or of the product of intensities and areas of pro- and active forms when present, as previously described by Baugh et al. ${ }^{16}$

\section{Cell Isolation Procedures and Functional Experiments}

When available, up to five biopsies from patients and controls were collected in sterile medium (PBS calcium- and magnesium-free, supplemented with $100 \mathrm{U} / \mathrm{ml}$ of penicillin and $100 \mu \mathrm{g} / \mathrm{ml}$ of streptomycin). The epithelial layer was removed by using 1-mmol/l ethylene diamine tetra-acetic acid and 1-mmol/l dithiothreitol (both from Sigma). After continuous agitation for $1 \mathrm{~h}$ at $37^{\circ} \mathrm{C}$, the supernatant containing epithelial cells was eliminated and the remaining tissue washed twice with RPMI-1640 medium and treated with $128 \mathrm{U} / \mathrm{ml}$ type $1 \mathrm{~A}$ collagenase (Sigma) for $2 \mathrm{~h}$ with continuous agitation at $37^{\circ} \mathrm{C}$. The crude cell suspension was then allowed to stand for $5 \mathrm{~min}$ to permit sedimentation of debris. The supernatant containing the LPMCs was washed twice in RPMI-1640 medium and the viability of the single cell suspension was determined by trypan blue exclusion. Cells were not used if viability did not exceed $90 \%$. Phenotypical characterization of LPMCs was performed by FACScan flow cytometer (Becton Dickinson, UK) by using the following 
reagents and antibodies: TriTEST CD3 FITC/CD8 PE/CD45 PerCP, TriTEST CD3 FITC/CD4 PE/CD45 PerCP, TriTEST CD3 FITC/CD19 PE/CD45 PerCP, TriTEST CD3 FITC/CD16 + CD56 PE/CD45 PerCP, anti-CD14 monoclonal antibody and anti-CD66 monoclonal antibody (all from Becton Dickinson Biosciences San Jose, CA, USA).

Primary cultures of myofibroblasts were obtained by following the method of Jobson et $a l .{ }^{17}$ Briefly, samples of human adult normal jejunal mucosa harvested from three patients undergoing gastrectomy for carcinoma were separated from the underlying muscularis mucosae by microdissection. $\mathrm{Mu}-$ cosal strips were subsequently denuded of epithelial cells by treatment with 1-mmol/l ethylene diamine tetra-acetic acid and 1-mmol/l dithiothreitol (both from Sigma), and put on Dulbecco's-modified eagle whole growth medium containing 15\% fetal calf serum, glutamine $(2 \mathrm{mM})$, penicillin $\mathrm{G}(100 \mathrm{U} / \mathrm{ml})$, streptomycin $(100 \mu \mathrm{g} / \mathrm{ml})$ and amphotericin-B $(0.25 \mu \mathrm{g} / \mathrm{ml})$. At the beginning, LPMCs migrated via basement membrane pores and were removed by frequent changes of the medium; colonies of myofibroblasts then appeared after at least 2 weeks. At this stage, the strips were removed, and the colonies grew to form a confluent monolayer over the following 1-3 weeks. Characterization of myofibroblasts was carried out by immunocytochemistry using a mouse antiactin smooth muscle monoclonal antibody (Chemicon International, Inc., Temecula, CA, USA; at 1:1000 dilution).

Both LPMCs and myofibroblasts were incubated at $37^{\circ} \mathrm{C}$ for $48 \mathrm{~h}$ in the presence or in the absence of IFN- $\gamma$ (R\&D System, Minneapolis, MN, USA) at a concentration of $1000 \mathrm{U} / \mathrm{ml}$, or TNF- $\alpha$ (R\&D System) at $50 \mathrm{ng} / \mathrm{ml}$. At the end of the incubation, LPMC suspensions were pipetted up and down to allow adherent cells to be removed and centrifuged. All pellets were washed in PBS and frozen at $-80^{\circ} \mathrm{C}$ until RNA extraction, whereas myofibroblasts were harvested before centrifugation after a 5-10 min

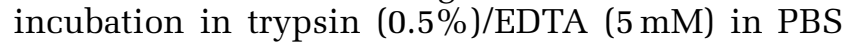
at $37^{\circ} \mathrm{C}$ followed by addition of whole Dulbecco'smodified eagle growth medium.

\section{Mucosal Morphometry}

In order to quantify the degree of villous flattening, we measured the surface area to volume ratio of duodenal mucosa by using a Weibel graticule (Graticules Ltd, Tunbridge Wells, UK) inserted in the microscope eyepiece, according to the method of Dunnill and Whitehead, ${ }^{18}$ standardized by Corazza et $a .^{19} \mathrm{~A}$ record was made of the number of times the lines cut the mucosal surface $(c)$ and the number of hits $(h)$, that is, end points of the lines falling on the lamina propria. At a constant magnification $(\times 125)$, the length $(I)$ of each line of the graticule was $1.7 \times 10^{-2} \mathrm{~cm}$. The ratio $c / l \times h$ was then calculated and regarded as a measure of surface area to volume ratio.

\section{Statistical Analysis}

Statistical comparisons between median values were performed using the Mann-Whitney $U$ test for nonparametric data. The correlations were examined by using the Spearman's rank correlation test. A level of $P$ less than 0.05 was considered statistically significant.

\section{Results}

\section{Mucosal Morphometry}

The quantitative assessment of the surface area to volume ratio carried out in each patient and control showed that this value was significantly lower (median 9.3, range 5.8-14.6) in active CD compared to treated CD (median 33.0, range 27.9-41.3, $P<0.001$ ) and controls (median 35.2 , range $31.5-$ 44.3, $P<0.001$ ), indicating the presence of villous flattening. No significant difference was found between treated CD and controls.

\section{MMP and Cytokine mRNA Transcript Levels}

Quantitative RT-PCR carried out on duodenal mucosal samples revealed that MMP-1 and MMP-12 were significantly increased in untreated CD in comparison to treated CD $(P=0.002$ and $P=0.0004$, respectively) and controls $(P=0.0012$ and $P=0.0003$, respectively), whereas MMP-3 and MMP-9 did not show any difference between the three groups (Figure 1). Furthermore, in both untreated and treated CD patients, MMP-2 transcript was significantly ( $P=0.0133$ and $P=0.0142$, respectively) reduced when compared to control mucosa, whereas MMP14 appeared similarly decreased only in treated CD with respect to controls $(P=0.0078)$ (Figure 1). The analysis of TIMP-1 transcript levels revealed a significant upregulation in active disease, being increased two-fold in comparison to treated disease and controls $(P=0.0012$ and $P=0.0311$, respectively) (Figure 2). As regards Th-1 proinflammatory cytokines, mucosal samples of untreated patients expressed statistically higher levels of IFN- $\gamma$ mRNA when compared to those found in patients on glutenfree diet and controls $(P=0.0121$ and $P=0.01002$, respectively), whereas TNF- $\alpha$ mRNA turned out to be suppressed ( $P=0.0001$ for both) (Figure 2). Furthermore, in active CD, MMP-12 mRNA directly correlated with IFN- $\gamma$ mRNA levels $\left(r_{\mathrm{s}}=0.69, P<0.05\right)$ and inversely with the degree of villous atrophy expressed as surface area to volume ratio $\left(r_{\mathrm{s}}=-0.70\right.$, $P<0.05)$. No other significant correlation was found between MMP levels and the other parameters.

\section{MMP Zymography}

Gelatin zymography disclosed that pro-MMP-9 was upregulated two-fold in active CD in comparison to 

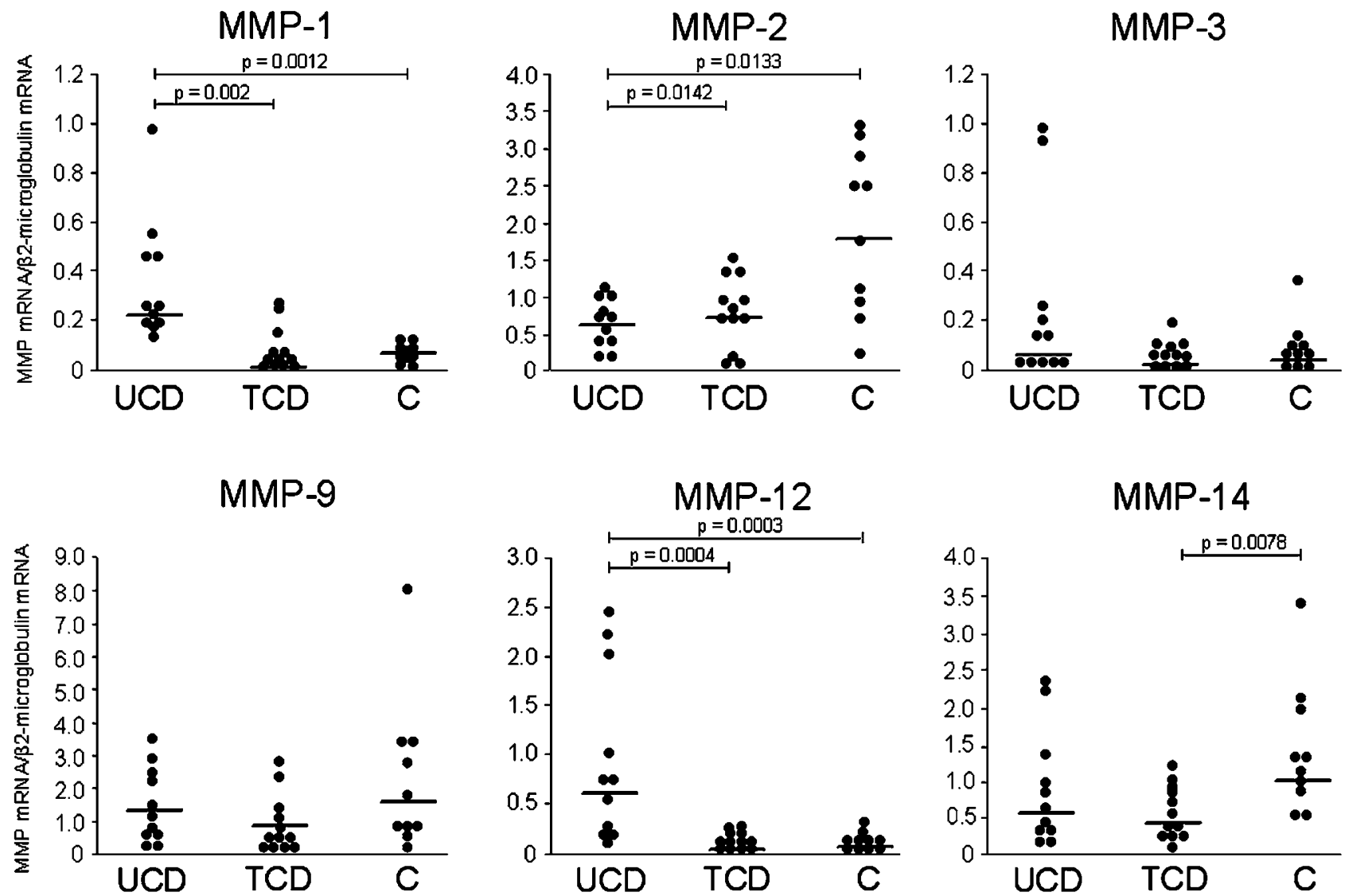

Figure 1 Matrix metalloproteinase (MMP) mRNA expression in duodenal mucosal samples from 11 untreated celiac disease (UCD) patients, 12 treated celiac disease (TCD) patients and 10 controls (C) as determined by real-time quantitative RT-PCR. Data are expressed as arbitrary units defined from the curve obtained by parallel RT-PCR analyses of serial dilutions of the standard. One unit was defined as the amount of the mRNA species present in $1 \mathrm{pg}$ of the standard. To compare mRNA levels of different samples, results were standardized to mRNA levels of the constitutive $\beta 2$-microglobulin. Horizontal bars indicate medians.

TIMP-1

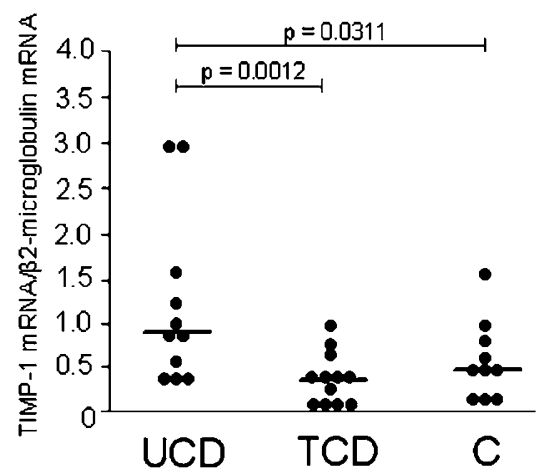

IFN- $\gamma$

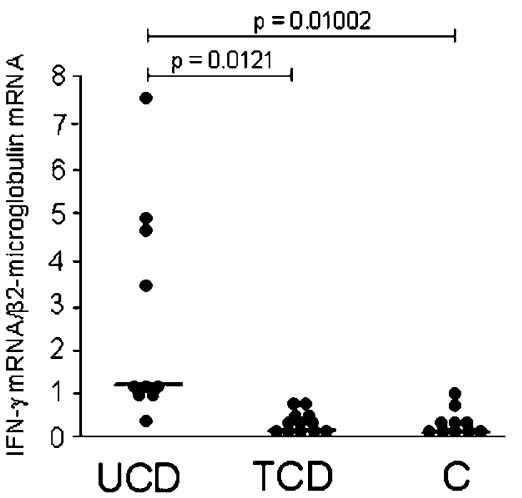

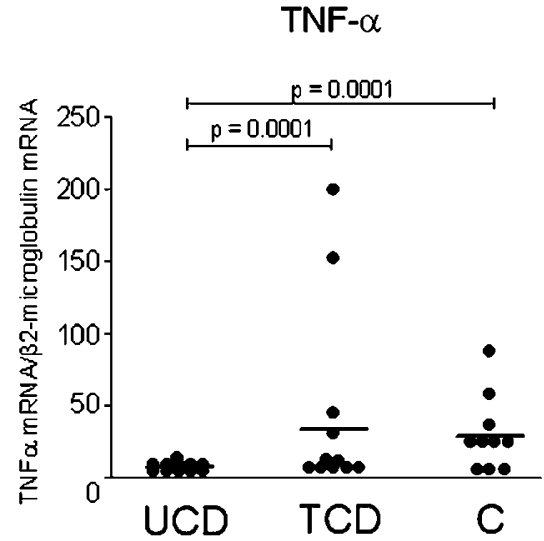

Figure 2 Transcript levels of tissue inhibitor of MMPs (TIMP-1), IFN- $\gamma$, and TNF- $\alpha$ in duodenal mucosal samples from 11 untreated celiac disease (UCD) patients, 12 treated celiac disease (TCD) patients and 10 controls (C) as determined by real-time quantitative RT-PCR. Data were normalized to $\beta 2$-microglobulin RNA as described for Figure 1. Horizontal bars indicate medians.

control and treated CD mucosa (Figure 3a). In contrast, MMP-2, determined as both pro- and active enzyme, showed a value that was about double in controls with respect to that found in untreated patients, while in treated patients the level was almost undetectable (Figure 3a). Two additional bands of Mws 80 and $38 \mathrm{kDa}$, prevalent in patients following a gluten-free diet and controls, respectively, did not disappear with 1,10-phenantroline treatment (data not shown) and cannot therefore be 
attributed to any MMP. Even though densitometry may underestimate the MMP level in cases of high concentrations, because of the complete removal of the substrate ${ }^{16}$ the quantitative assessment of the bands performed in all samples confirmed these results either in terms of band intensity or area (Figure 3b).

The evaluation of the caseinolytic activity, which can be attributed to MMP-1 and MMP-3 without differentiation between these enzymes because of overlapping $\mathrm{Mw}$, revealed a significant two-fold increase for both pro- and active forms in untreated patients compared to controls, while treated CD patients showed a weak presence of the active enzyme being the proenzyme almost undetectable (Figure 4a). The densitometric analysis of both band intensity and area performed in all samples confirmed these data (Figure 4b). As was evident, the gluten-free diet led to a considerable reduction of caseinolytic activity when compared to active disease samples.
Finally, the elastolytic activity was found strongly upregulated by about four-fold in active $\mathrm{CD}$ in comparison to the very low levels found in treated $\mathrm{CD}$ and controls mainly as concerns the active form of the enzyme (Figure 5a), as was more evident by densitometric quantification (Figure 5b).

\section{Functional Experiments}

The characterization of stromal cells isolated from normal jejunum demonstrated anti-smooth muscle positivity (data not shown), defining these cells as myofibroblasts. The characterization of LPMCs is given in Table 2. Notably, in both treated and untreated CD mucosa, we found a significant increase of $\mathrm{CD}^{+}$and $\mathrm{CD} 14^{+}$cell percentages in comparison to normal mucosa, the latter showing a significantly higher percentage of $\mathrm{CD}^{+}$and CD16/ $56^{+}$cells when compared to the other two groups, while no differences were found concerning the

a

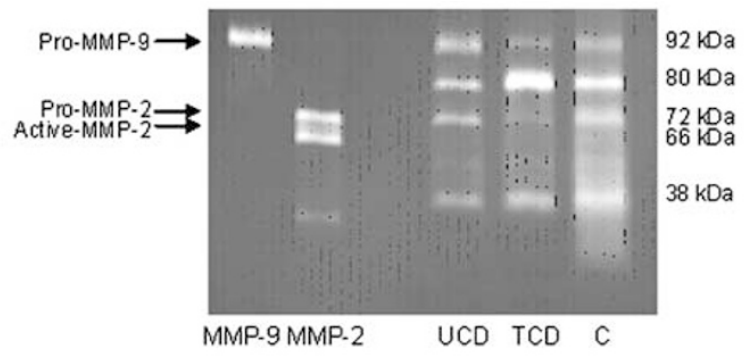

b

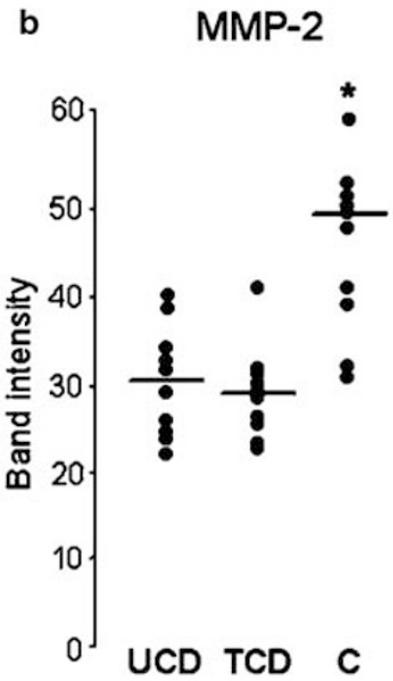

Pro-MMP-9

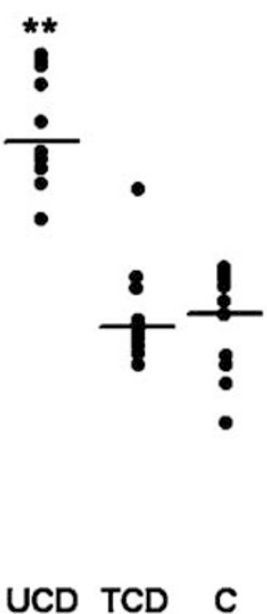

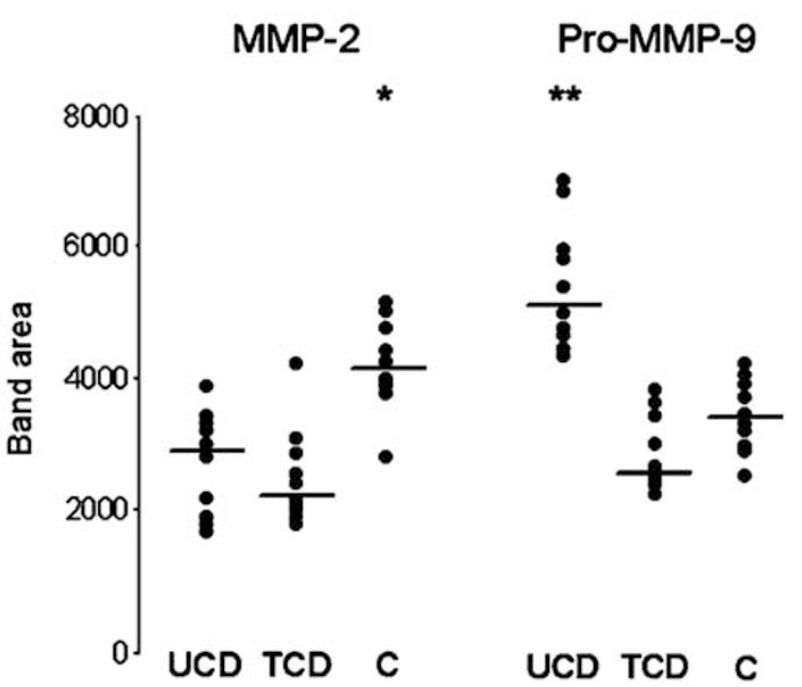

Figure 3 (a) Gelatinolytic activity of duodenal mucosal samples from one representative untreated celiac (UCD) patient, one representative treated celiac (TCD) patient and one representative control (C) subject, and of MMP-2 and MMP-9 standards. Cleared bands indicate areas of enzymatic activity. On the right side, the relative Mw are indicated in kilodaltons (kDa). On the left side, the corresponding enzyme as pro- and active-forms are shown. The bands of 80 and $38 \mathrm{kDa}$, which did not disappear with 1,10phenantroline treatment (data not shown), cannot be attributed to any MMP. (b) Quantification of enzyme activity in UCD, TCD and control groups was performed by scanning densitometry and is expressed as intensity and surface area for the pro-MMP-9 band, and as the sum of intensities and areas of bands attributable to pro-MMP-2 and active MMP-2. Horizontal bars represent the median value. ${ }^{*} \mathrm{P}<0.05$ vs TCD and UCD; ${ }^{*} P<0.001$ vs TCD and C. 

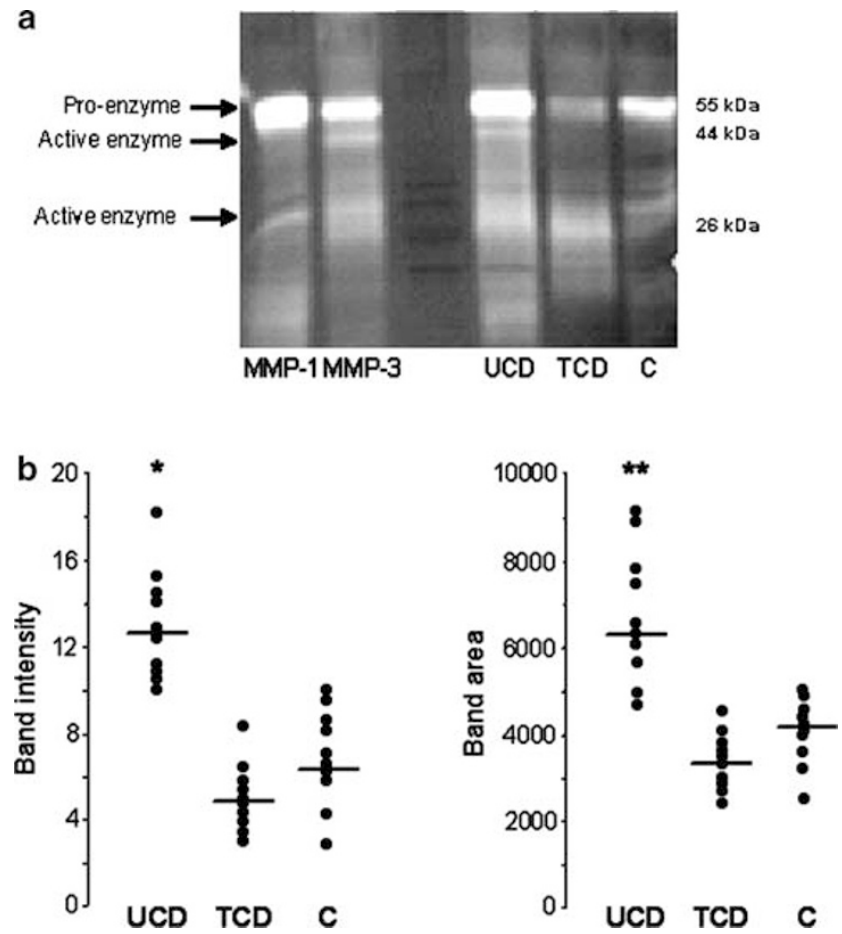

Figure 4 (a) Caseinolytic activity of duodenal mucosal samples from one representative untreated celiac (UCD) patient, one representative treated celiac (TCD) patient and one representative control (C) subject, and of MMP-1 and MMP-3 standards. Cleared bands indicate areas of enzymatic activity. Relative $\mathrm{Mw}$ in kilodaltons $(\mathrm{kDa})$ on the right side and the corresponding proand active-forms of the enzymes on the left side are shown. (b) Quantification of enzyme activity in UCD, TCD and control groups was performed by scanning densitometry and expressed as the sum of intensities and areas of bands attributable to pro- and active MMP-1/3. Horizontal bars represent the median value. ${ }^{*} P<0.001$ vs TCD and $\mathrm{C} ;{ }^{*} P<0.01$ vs TCD and C.

total number of $\mathrm{CD}^{+}, \mathrm{CD} 19^{+}$and $\mathrm{CD}^{+} 6^{+}$cell percentages.

To determine whether Th-1 proinflammatory cytokines are able to modulate the expression of MMPs and TIMP-1 by intestinal myofibroblasts and LPMCs, cultures of these two cell populations were incubated in the absence or presence of IFN- $\gamma$ and TNF- $\alpha$. As shown in Figure 6a, unstimulated myofibroblasts obtained from normal jejunal mucosa produced very high levels of MMP-2 mRNA, and smaller amounts of MMP-1, MMP-3 and MMP-14, whereas MMP-9 and MMP-12 were almost undetectable. While stimulation by IFN- $\gamma$ inhibited MMP-1, MMP-2, MMP-3 and MMP-14 mRNAs and had no effect on MMP-9 and MMP-12, TNF- $\alpha$ slightly increased only MMP-3 and MMP-14. As regards TIMP-1, we found that TNF- $\alpha$ treatment appeared unable to modify the basal level, whereas IFN- $\gamma$ determined only a slight decrease (data not shown).

The amounts of MMPs produced by unstimulated and stimulated LPMCs differed markedly in the three groups studied, as shown in Figure 6b. When
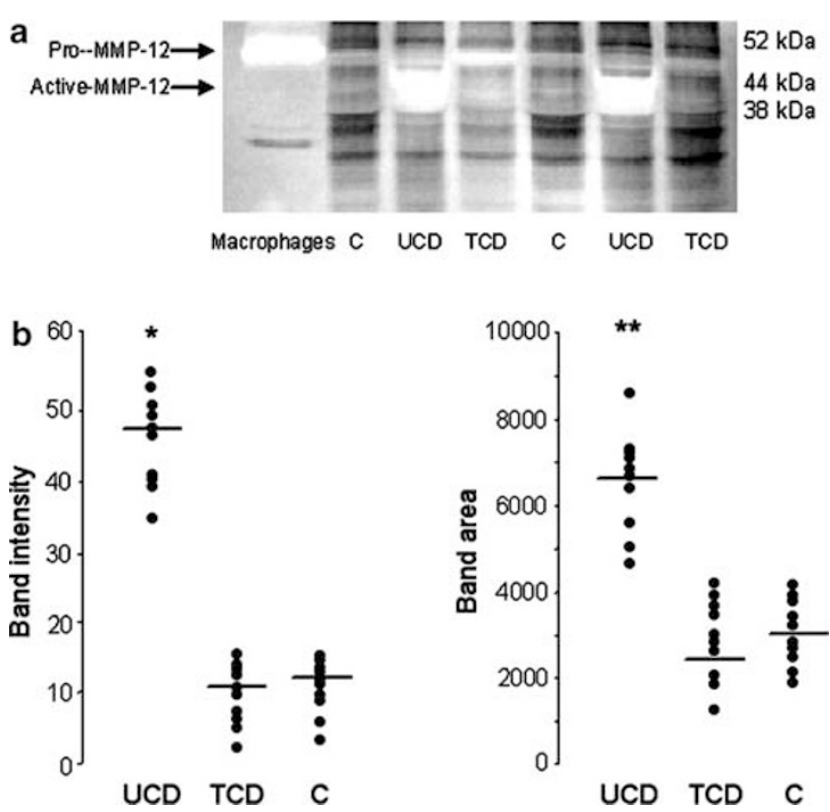

Figure 5 (a) Elastolytic activity of duodenal mucosal samples from two representative untreated celiac (UCD) patients, two representative treated celiac (TCD) patients and two representative control (C) subjects, and of supernatant of human macrophages cultured in the presence of granulocyte macrophage colony stimulating factor as MMP-12 positive control. Cleared bands indicate areas of enzymatic activity. Relative $\mathrm{Mw}$ in kilodaltons $(\mathrm{kDa})$ on the right side and the corresponding proand active-forms of the enzyme on the left side are shown. (b) Quantification of enzyme activity in UCD, TCD and control groups was performed by scanning densitometry and expressed as the sum of intensities and areas of the bands attributable to proand active MMP-12. Horizontal bars represent the median value. ${ }^{*} P<0.001$ vs TCD and $\mathrm{C} ;{ }^{*} P<0.01$ vs TCD and C.

LPMCs were isolated from untreated CD mucosa, the basal pattern was strongly dominated by MMP-9 and above all by MMP-12. This pattern was not further modified by the addition of both cytokines with the exception of a significant reduction of MMP-3 operated by IFN- $\gamma$ and of MMP-12 by TNF- $\alpha$. In LPMCs isolated from treated CD mucosa, undetectable levels of MMP-1, MMP-9 and MMP-12 and very low levels of MMP-2, MMP-3 and MMP-14 were observed in basal conditions, while IFN- $\gamma$ was able to induce MMP-1 and MMP-12 mRNA expression, and TNF- $\alpha$ stimulated that of MMP-9. When LPMCs were isolated from normal mucosa, only low levels of MMP-9 mRNA were found in basal condition, whereas TNF- $\alpha$ was able to induce the expression of all MMPs, with the exception of MMP-12, which was exclusively upregulated by IFN- $\gamma$. Finally, as regards TIMP-1 transcript level, we found that whereas in normal and treated CD mucosal samples, both cytokines were able to increase its expression, in active CD mucosa, there was the presence of a high basal level that was unaffected by the treatment with both cytokines (data not shown). 


\section{Discussion}

Modulation of cell-matrix interaction occurs through the action of MMPs, a complex proteolytic system, responsible for hydrolysis of a variety of ECM components. Signals from the ECM regulate proliferation, differentiation and apoptosis of epithelial and other cells, exemplifying how central ECM synthesizing and degrading cells are for the maintenance of mucosal homeostasis. ${ }^{20}$ In addition, immunologically mediated cascades that give rise to the release of MMPs seem to play a central role in the pathogenesis of inflammatory bowel diseases. ${ }^{21}$ However, while the role of MMPs in Crohn's disease, ulcerative colitis and pouchitis is well established, ${ }^{16,22,23}$ less is known about their relevance in CD. By using in situ hybridization, Daum et $a l^{11}$ were the first to describe the presence of a high number of $\mathrm{MMP}-1^{+}$cells in both the subepithelial region and the lamina propria, while MMP- $3^{+}$and TIMP-1 ${ }^{+}$cells turned out to be increased only in the subepithelial region of untreated CD. Subsequently, using the same technique, Salmela et $a l^{12}$ demonstrated a significant increase of $\mathrm{MMP}^{+}{ }^{+}$and $\mathrm{MMP}-12^{+}$cells in dermal and duodenal lesions of patients affected by dermatitis herpetiformis.

By quantifying MMP transcripts by means of realtime RT-PCR, we confirmed raised levels of MMP-1, MMP-12 and TIMP-1 in untreated CD and described for the first time a suppression of MMP-2, which appears to be the prevalent proteolytic enzyme in normal intestinal mucosal and almost exclusively produced by unstimulated myofibroblasts, in accordance with a previous report. ${ }^{24}$ Furthermore, we found unmodified levels of MMP-3 in CD and decreased value of MMP-14 in treated CD in comparison to normal mucosal. Since MMPs are subjected to a strict control at both transcriptional and post-transcriptional levels, ${ }^{8,9}$ we also investigated their activity by using gel zymography. Our findings demonstrated that caseinolytic, gelatinolytic and elastolytic levels are upregulated in active CD. More in depth, the increased caseinolytic expression is likely attributable to MMP-1, whose mRNA transcripts were significantly raised in untreated CD patients, while as concerns MMP-9, the apparent discrepancy between its unchanged mRNA level despite increased gelatinolytic expression suggests a mainly post-transcriptional upregulation. Moreover, the presence of two additional bands with gelatinolytic activity not imputable to MMP-2 or MMP-9 suggests that proteases other than MMPs, such as serine proteases, ${ }^{25}$ may be responsible for matrix ECM turnover in the investigated specimens. However, the more impressive result was the high elastolytic activity due to the active enzyme found in untreated CD.

In treated CD mucosa, we observed a peculiar MMP mRNA profile that resembled that of normal mucosa as regards MMP-1 and MMP-12, and that of 


\section{CONTROL MYOFIBROBLASTS}

\section{LAMINA PROPRIA MONONUCLEAR CELLS}
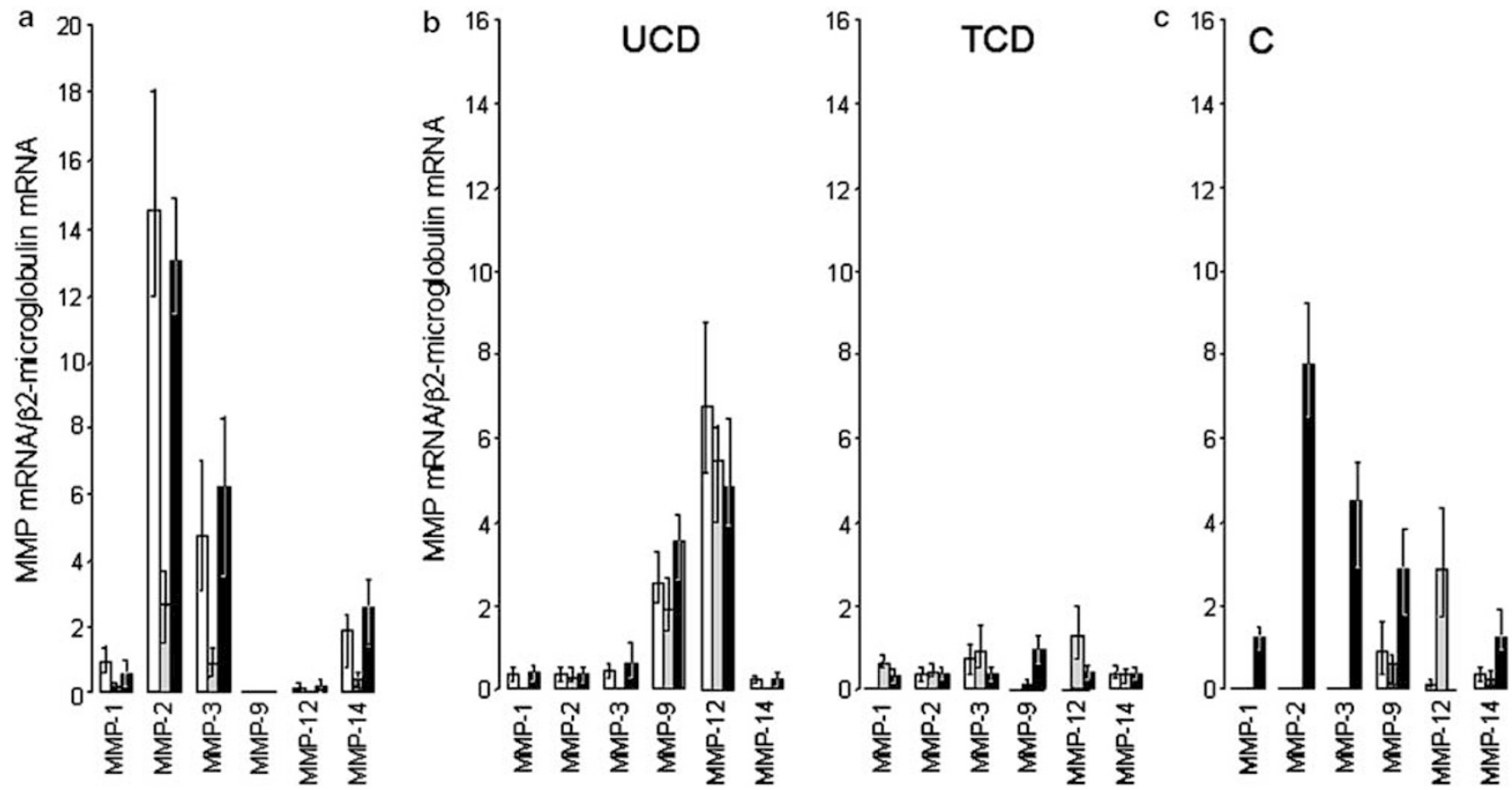

Figure 6 Real-time quantitative RT-PCR for matrix metalloproteinase (MMP) mRNA in small bowel myofibroblasts isolated from surgical specimens (a) and in freshly isolated duodenal LPMCs (b) from mucosal samples of four untreated celiac disease (UCD) patients, four treated celiac disease (TCD) patients and four controls (C), incubated in the absence (white bars) or presence of IFN- $\gamma$ (grey bars) or TNF- $\alpha$ (black bars). Data are expressed as arbitrary units derived from parallel RT-PCR analysis of serial dilutions of the standard. One unit was defined as the amount of mRNA species present in $1 \mathrm{pg}$ of the standard. To compare mRNA levels in different samples, results were standardized with respect to $\beta 2$-microglobulin. Results represent the median value (range) of four different experiments performed in triplicates.

active CD as regards MMP-2 and MMP-14. The overall MMP pattern, with fairly low transcript levels of all MMPs investigated, is indicative of a mucosa in a quiescent state.

Since experimental models of mucosal enteropathy suggest that tissue injury is mediated by MMPs produced by stimulated residential stromal and immune cells, ${ }^{10}$ we tried to correlate the MMP pattern of active CD with the degree of mucosal damage. The demonstration of a significant correlation between the levels of MMP-12 and the degree of villous atrophy points to a pathogenetic relevance of this enzyme. Since a correlation between the magnitude of villous atrophy and that of enterocyte apoptosis has been shown previously in untreated CD patients, ${ }^{26}$ it is conceivable that, by degrading either interstitial ECM or basement membranes, ${ }^{12}$ MMP-12 ultimately leads to the collapse of villous architecture and subsequent enterocyte shedding to the lumen. Furthermore, since MMP expression is highly dependent on cytokines ${ }^{27,28}$ and gluten exposure in patients with CD rapidly elicits high levels of Th-1 cytokines, such as IFN- $\gamma$, IL-2 and TNF- $\alpha,{ }^{13,14}$ we simultaneously determined the levels of IFN- $\gamma$ and TNF- $\alpha$. Our results confirm a strong upregulation of IFN- $\gamma$ in active CD but not of TNF- $\alpha$, the latter being in accordance with recent findings by Forsberg et $a l^{29}$ who showed a suppression of TNF- $\alpha$ mRNA in this condition. This discrepancy might be a consequence of the different technique used by Kontakou et $a l^{13}$ that allows only a semiquantitative assessment. In this light, the significant positive correlation between IFN- $\gamma$ and MMP-12 transcript levels suggests a crucial role of this cytokine in upregulating MMP-12 production.

The strong influence of proinflammatory cytokines on MMP expression was clearly demonstrated by our functional experiments carried out on isolated cells from small intestinal mucosa. In physiological conditions, unstimulated LPMCs produced very small amounts of MMPs while they appeared to be highly sensitive to IFN- $\gamma$ as regards MMP-12 production and to TNF- $\alpha$ as regards the other MMPs, supporting the role played by these cells in generating mucosal injury. By contrast, LPMCs purified from active CD basally produced high levels of MMP-9 and MMP-12, irrespective of additional cytokine stimulation, a behaviour that might be explained by a pre-existing cytokine hyperstimulation that disables these cells from responding to a further stimulation. This assumption fits well with the increased gelatinolytic and elastolytic activities observed in this condition. In treated CD again, LPMCs showed a unique 
proteolytic pattern characterized by an almost total absence of MMP expression in basal conditions like normal mucosa, and by a weak response to proinflammatory cytokines as found in active CD mucosa. One explanation might be the presence of the same cellular phenotype in the celiac mucosa regardless of the state of activation and characterized by an increase of $\mathrm{CD}^{+} \mathrm{T}$ lymphocytes and monocyte/ macrophage population, whereas $\mathrm{CD}^{+}{ }^{+} \mathrm{T}$ lymphocytes and natural killer population appeared reduced. Furthermore, the finding that TNF- $\alpha$ is able to stimulate mainly the MMP-3 and MMP-9 expression indicates that this cytokine is a key inducer of a destructive pattern, the MMP-3 presence always being associated with ulcer formation, ${ }^{30}$ whereas IFN- $\gamma$ appears to primarily induce a remodelling pattern.

The results of the present study are in line with those produced by MacDonald's group who used human fetal small intestine as an experimental model of gut inflammation ${ }^{31-33}$ and demonstrated that activation of lamina propria lymphocytes with pokeweed mitogen or anti-CD3 plus IL-12 led to a marked increase of MMP production strongly dominated by MMP-3 resulting in severe tissue injury resembling Crohn's disease. ${ }^{10,34}$ On the contrary, their activation with the superantigen staphylococcal enterotoxin B or anti-CD3 plus IFN- $\alpha$ caused less cytokine production, less MMP activation and tissue damage mainly represented by villous atrophy and crypt hyperplasia. ${ }^{35,36}$ Accordingly, it can be speculated that the two main Th1-mediated diseases of the human small intestine, that is, CD and Crohn's disease, are characterized by different cytokine and MMP profiles which ultimately result in two different types of mucosal injury. In the case of $\mathrm{CD}$, activation of lamina propria $\mathrm{T}$ cells by tissue transglutaminase-modified gliadin epitopes elicits a high production of IFN- $\gamma$ which in turn causes a strong upregulation of MMP-12 able to induce villous atrophy and crypt hyperplasia. In the case of Crohn's disease, activation of lamina propria $\mathrm{T}$ cells by bacterial antigens results in a high production of TNF- $\alpha$, which in turn causes an overproduction of MMP-3, which leads to the characteristic transmural lesions.

In conclusion, our data are consistent with the presence of a characteristic mucosal MMP pattern in active CD highly dominated by MMP-12. This enzyme may be considered one of the final steps in the cascade of immunological events leading to tissue injury. The recently described lack of association of MMP-1 and MMP-3 genes with $\mathrm{CD}^{37}$ points to a crucial role of mucosal regulation of MMP. IFN- $\gamma$ appears to be the culprit of the observed MMP-12 upregulation and confirmation of its relevance in CD pathogenesis has been indirectly provided by the finding that anti-IFN- $\gamma$ antibodies can prevent villous atrophy both in CD mucosal samples treated with the supernatants of gliadin-activated T-cell clones $^{38}$ and in murine graft-vs-host-disease, ${ }^{39}$ a condition that shares many features in common with CD.

\section{Acknowledgements}

We wish to acknowledge the German Research Foundation DGF Grant Schu 646/11-2\&11-3, the European Union Grant QLRT-1999-00037, and the German Celiac Foundation for financial support.

\section{References}

1 Arentz-Hansen H, McAdam SN, Molberg Ø, et al. Celiac lesion $\mathrm{T}$ cells recognize epitopes that cluster in regions of gliadins rich in proline residues. Gastroenterology 2002;123:803-809.

2 Vader W, Kooy Y, Van Veelen P, et al. The gluten response in children with celiac disease is directed toward multiple gliadin and glutenin peptides. Gastroenterology 2002;122:1729-1737.

3 Sollid LM. Molecular basis of celiac disease. Annu Rev Immunol 2000;18:53-81.

4 Dieterich W, Ehnis T, Bauer M, et al. Identification of tissue transglutaminase as the autoantigen of celiac disease. Nat Med 1997;3:797-801.

5 Martucci S, Corazza GR. Spreading and focusing of gluten epitopes in celiac disease. Gastroenterology 2002;122:2072-2075.

6 Shan L, Molberg Ø, Parrot I, et al. Structural basis for gluten intolerance in celiac sprue. Science 2002;297: 2275-2279.

7 MacDonald TT, Spencer J. Evidence that activated mucosal $\mathrm{T}$ cells play a role in the pathogenesis of enteropathy in human small intestine. J Exp Med 1988;16:1341-1349.

8 Goetzl EJ, Banda MJ, Leppert D. Matrix metalloproteinases in immunity. J Immunol 1996;156:1-4.

9 Nagase H, Woessner JF. Matrix metalloproteinases. J Biol Chem 1999;274:21491-21494.

10 Pender SL, Tickle SP, Docherty AJ, et al. A major role for matrix metalloproteinases in $\mathrm{T}$ cell injury in the gut. J Immunol 1997;158:1582-1590.

11 Daum S, Bauer U, Foss H-D, et al. Increased expression of mRNA for matrix metalloproteinases-1 and -3 and tissue inhibitor of metalloproteinases-1 in intestinal biopsy specimens from patients with coeliac disease. Gut 1999;44:17-25.

12 Salmela MT, Pender S-F, Reunala T, et al. Parallel expression of macrophage metalloelastase (MMP-12) in duodenal and skin lesions of patients with dermatitis herpetiformis. Gut 2001;48:496-502.

13 Kontakou M, Przemioslo RT, Sturgess RP, et al. Cytokine mRNA expression in the mucosa of treated celiac patients after wheat peptide challenge. Gut 1995;37:53-57.

14 Nilsen EM, Jahnsen FL, Lundin KE, et al. Gluten induces an intestinal cytokine response strongly mediated by interferon gamma in patients with celiac disease. Gastroenterology 1998;115:551-563.

15 Thogeisson UP, Mackay AR. Characterization of metastatic tumor cell. In: Gallegner CT, Rees RC, Reynolds CW (eds). Tumor Immunology: A Practical Approach. University Press: Oxford, UK, 1992, pp 82-90. 
16 Baugh MD, Perry MJ, Hollander AP, et al. Matrix metalloproteinase levels are elevated in inflammatory bowel disease. Gastroenterology 1999;117:814-822.

17 Jobson TM, Billington CK, Hall IP. Regulation of proliferation of human colonic subepithelial myofibroblasts by mediators important in intestinal inflammation. J Clin Invest 1998;101:2650-2657.

18 Dunnil MS, Whitehead R. A method for the quantitation of small intestinal biopsy specimens. J Clin Pathol 1972;25:243-246.

19 Corazza GR, Frazzoni M, Dixon MF, et al. Quantitative assessment of the mucosal architecture of jejunal biopsy specimens: a comparison between linear measurement, stereology, and computer aided microscopy. J Clin Pathol 1985;38:765-770.

$20 \mathrm{Vu}$ TH, Werb Z. Matrix metalloproteinases: effectors of development and normal physiology. Genes Dev 2000;14:2123-2133.

21 MacDonald TT, Bajaj-Elliott M, Pender SL. T cells orchestrate intestinal mucosal shape and integrity. Immunol Today 1999;20:505-510.

22 von Lampe B, Barthel B, Coupland SE, et al. Differential expression of matrix metalloproteinases and their tissue inhibitors in colon mucosa of patients with inflammatory bowel disease. Gut 2000;47:63-73.

23 Stallmach A, Chan CC, Ecker KW, et al. Comparable expression of matrix metalloproteinases 1 and 2 in pouchitis and ulcerative colitis. Gut 2000;47:415-422.

24 Baugh MD, Hollander AP, Evans GS. The regulation of matrix metalloproteinase production in human colonic fibroblasts. Ann N Y Acad Sci 1998;859:175-179.

25 Ghersi G, Dong H, Goldstein LA, et al. Regulation of fibroblast migration on collagenous matrix by a cell surface peptidase complex. J Biol Chem 2002;277: 29231-29241.

26 Ciccocioppo R, Di Sabatino A, Parroni R, et al. Increased enterocyte apoptosis and Fas-Fas Ligand system in celiac disease. Am J Clin Pathol 2001;115: 494-503.

27 Qin H, Moellinger JD, Wells A, et al. Transcriptional suppression of matrix metalloproteinase-2 gene expression in human astroglioma cells by TNF- $\alpha$ and IFN- $\gamma$. J Immunol 1998;161:6664-6673.

28 Lewis M, Amento EP, Unemori EN. Transcriptional inhibition of stromelysin by interferon- $\gamma$ in normal human fibroblasts is mediated by the AP-1 domain. J Cell Biochem 1999;72:373-386.

29 Forsberg G, Hernell O, Melgar S, et al. Paradoxical coexpression of proinflammatory and down-regulatory cytokines in intestinal $\mathrm{T}$ cells in childhood celiac disease. Gastroenterology 2002;123:667-678.

30 Saarialho-Kere UK, Vaalamo M, Puolakkainen $\mathrm{P}$, et al. Enhanced expression of matrilysin, collagenase, and stromelysin-1 in gastrointestinal ulcers. Am J Pathol 1996;148:519-526.

31 MacDonald TT, Spencer J. Evidence that activated mucosal $\mathrm{T}$ cells plays a role in the pathogenesis of enteropathy in human small intestine. J Exp Med 1988;167:1341-1349.

32 Lionetti P, Breese E, Braegger CP, et al. T cell activation can induce either mucosal destruction or adaptation in cultured human fetal small intestine. Gastroenterology 1993;105:373-381.

33 Pender S-F, Lionetti P, Murch SH, et al. Proteolytic degradation of intestinal mucosal extracellular matrix after lamina propria $\mathrm{T}$ cell activation. Gut 1996;39: 284-290.

34 Monteleone G, MacDonald TT, Wathen NC, et al. Enhancing lamina propria Th1 cell responses with interleukin 12 produces severe tissue injury. Gastroenterology 1999;117:1069-1077.

35 Lionetti P, Spencer J, Breese EJ, et al. Activation of mucosal $\mathrm{V}$ beta $3+\mathrm{T}$ cells and tissue damage in human small intestine by the bacterial superantigen, Staphylococcus aureus enterotoxin B. Eur J Immunol 1993;23: 664-668.

36 Monteleone G, Pender S-F, Wathen NC, et al. Interferon- $\alpha$ drives $\mathrm{T}$ cell-mediated immunopathology in the intestine. Eur J Immunol 2001;31:2247-2255.

37 Louka AS, Stensby EK, Ek J, et al. Coeliac disease candidate genes: no association with functional polymorphisms in matrix metalloproteinase 1 and 3 gene promoters. Scand J Gastroenterol 2002;37:931-935.

38 Przemioslo RT, Lundin KE, Sollid LM, et al. Histological changes in small bowel mucosa induced by gliadin-sensitive $\mathrm{T}$ lymphocytes can be blocked by anti-interferon gamma antibody. Gut 1995;36:874-879.

39 Mowat AM. Antibodies to IFN- $\gamma$ prevent immunologically mediated intestinal damage in murine graftversus-host-reaction. Immunology 1989;68:18-23. 